\title{
A decade of outpatient antimicrobial use in older adults in Ontario: a descriptive study
}

\author{
Charlie Tan MD, Erin Graves MSc, Hong Lu PhD, Anna Chen MPH, Shudong Li PhD, \\ Kevin L. Schwartz MD MSc, Nick Daneman MD MSc
}

\section{Abstract}

Background: Antimicrobials are frequently prescribed to community-dwelling older adults. Our aim was to examine the prevalence, quantity and indications of antimicrobial prescriptions to older residents of Ontario.

Methods: We conducted a population-based analysis of outpatient antimicrobial prescriptions to residents of Ontario aged 65 years or more from 2006 to 2015. Antimicrobial prescriptions, infectious disease diagnoses and prescriber information were determined from linked health care databases. Our analyses were primarily focused on antibiotics, which account for most antimicrobial use.

Results: We identified 2879779 unique Ontario residents aged 65 years or more over our study period. On average, 40.7\% (range $40.1 \%-41.5 \%$ ) of older adult outpatients in any given year received 1 or more antibiotic prescriptions. Antibiotic use remained stable over the study period, averaging 25.1 (range 24.1-25.6) defined daily doses per 1000 person-days per year. Selection of antibiotics evolved, with increasing use of penicillins and decreasing use of fluoroquinolones and macrolides. For $65.7 \%$ of prescriptions, no infectious disease diagnoses were identified within 7 days of the prescription. Among prescriptions with an associated diagnosis, upper respiratory tract infection was most common (18.9\%), followed by urinary tract infection $(6.2 \%)$, skin/soft-tissue infection $(4.3 \%)$, lower respiratory tract infection $(4.2 \%)$ and other infection (1.2\%). Most antibiotics were prescribed by family physicians.

Interpretation: Antibiotic use among older adult outpatients in Ontario remained stable between 2006 and 2015. Current methods of measuring use are not capable of accurately determining indication, and, thus, additional data sources to monitor the appropriateness of community antimicrobial use are needed.

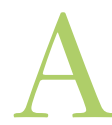

ntimicrobials are among the most commonly prescribed medications in Canada. Most are dispensed in an outpatient setting, accounting for $93 \%$ of total use in 2014. ${ }^{1}$ Many prescriptions are unnecessary or inappropriate, with antibiotics given for viral illnesses and increasing use of broad-spectrum agents. ${ }^{2-7}$ The evaluation of outpatient antimicrobial use is of pressing importance, as misuse is the primary driver of antimicrobial resistance. ${ }^{8}$ Patients prescribed antibiotics in primary care are more likely to develop antibiotic-resistant infections, ${ }^{9}$ and higher outpatient antimicrobial use has been associated with increased resistance, in both community and health care settings. ${ }^{10-14}$ Overuse is also associated with greater health care costs and more adverse events. ${ }^{15-17}$ Furthermore, the correlation between in-hospital antimicrobial use and resistance has been found to be poor, which suggests that outpatient prescribing drives much of inpatient resistance. ${ }^{18,19}$

Judicious use of antimicrobials is particularly important for older adults, who are prescribed these medications more fre- quently than younger people. ${ }^{1,20-23}$ Antimicrobials are the fourth most common drug class prescribed among older adults in Ontario. ${ }^{24}$ Older adults are at greater risk for adverse drug events than younger people $\mathrm{e}^{25,26}$ and have high rates of colonization with antimicrobial-resistant organisms. ${ }^{27-29}$

Several initiatives have raised awareness of inappropriate antimicrobial use and have promoted prudent prescribing. ${ }^{30,31}$ However, interventions for outpatient antimicrobial stewardship are challenging to implement, ${ }^{32}$ and surveillance of ambulatory prescribing in Canada has been limited despite calls to prioritize such efforts. ${ }^{33}$ The objective of

Competing interests: None declared.

This article has been peer reviewed.

Correspondence to: Nick Daneman, nick.daneman @sunnybrook.ca CMAJ Open 2017. DOI:10.9778/cmajo.20170100 
our study was to describe patterns of outpatient antimicrobial prescribing in older residents of Ontario from 2006 to 2015.

\section{Methods}

We conducted a province-wide analysis of antimicrobial prescriptions to older adults (aged $\geq 65 \mathrm{yr}$ ) in Ontario from Jan. 1, 2006, to Dec. 31, 2015.

\section{Data sources}

This study used population-based administrative databases housed at the Institute for Clinical Evaluative Sciences. These databases are well validated and have been used in studies on antimicrobial prescribing. ${ }^{34-38}$ The Ontario Drug Benefit Program database, which contains all publicly funded medications prescribed to Ontario residents aged 65 years or more, was used for information on antimicrobial prescribing. This database exhibits greater than $99 \%$ accuracy when compared against pharmacy dispensing data. ${ }^{34}$ To identify older adults and determine infectious disease diagnoses, the following databases were linked to the Ontario Drug Benefit Program database at the patient level: the Registered Persons Database, which contains demographic information for Ontario residents with publicly funded health insurance $(>95 \%$ of residents); the Ontario Health Insurance Plan database, which contains all billing claims made by health care providers for services performed in Ontario; the Discharge Abstracts Database, which contains information on all admissions, discharges and same-day surgical procedures in Ontario hospitals; and the National Ambulatory Care Reporting System, which contains information on all emergency department visits in Ontario hospitals.

\section{Antimicrobial prescriptions}

We used the Registered Persons Database to identify all Ontario residents aged 65 years or more as of Jan. 1 of each calendar year during our study period. Residents who had no contact with the health care system in the 7 years preceding assessment, or who died or moved out of province between calendar years were excluded. We then used the Ontario Drug Benefit Program database to determine the proportion of older adults who were prescribed an antimicrobial in each study year. Antimicrobials were classified into 1 of 4 categories: antibiotics, antivirals, antifungals and antiparasitics (Appendix 1, available at www.cmajopen.ca/content/5/4/ E878/suppl/DC1).

\section{Antibiotic prescriptions}

Our subsequent analyses were focused on antibiotics, as this is the most frequently prescribed category of antimicrobials and because resistance to antibiotics is of greatest public health concern. ${ }^{8}$ Using the Ontario Drug Benefit Program database, we determined the quantity of each antibiotic class and antibiotic drug prescribed to older adults in each study year. Antibiotics were grouped into 14 classes, with antitubercular agents removed from the analyses (Appendix 2, available at www.
cmajopen.ca/content/5/4/E878/suppl/DC1). Antibiotic use was measured in defined daily doses per 1000 person-days. Defined daily doses are a standardized metric of drug use based on an assumed average daily maintenance dosage..$^{39} \mathrm{We}$ calculated person-days as the total number of adults aged 65 years or more residing in Ontario in each study year multiplied by the number of days in that year.

Indications for antibiotic prescriptions: Each antibiotic prescription was subsequently linked to the physician claim, hospital discharge, same-day surgery and emergency department databases to identify any infectious disease diagnoses recorded within 7 days before or after the prescription. Diagnoses were grouped into the following categories: upper respiratory tract infection, lower respiratory tract infection, urinary tract infection, skin/soft-tissue infection, other infection and no recorded infection. We determined the proportion of use of each antibiotic class and antibiotic drug associated with each clinical indication.

Antibiotics prescribed for infectious disease diagnoses: We identified all infectious disease diagnoses recorded in the Ontario Health Insurance Plan database, Discharge Abstracts Database and National Ambulatory Care Reporting System for 2006 and 2015. These diagnoses were linked to the Ontario Drug Benefit Program database to examine whether a prescription for an antibiotic was filled in the 7 days before or after each diagnosis. We determined the overall numbers of antibiotic prescriptions as well as the 10 most commonly prescribed antibiotics for each diagnosis category.

\section{Responsible prescribers}

To determine the health care providers most responsible for outpatient antibiotic use among older adults, we determined the proportion of prescriptions, in individual claims and defined daily doses, attributable to family physicians and specialists. This analysis was conducted for 2006 and 2015.

\section{Statistical analyses}

Analyses were performed with SAS statistical software version 9.3 (SAS Institute Inc.) and R statistical software version 3.2.2 (R Foundation for Statistical Computing).

\section{Ethics approval}

Ethics approval was obtained from the Sunnybrook Health Sciences Centre Research Ethics Board.

\section{Results}

During the study period, 2879779 unique residents of Ontario aged 65 years or more were identified. The population of older adults increased from 1646909 in 2006 to 2176736 in 2015. On average, 40.7\% (range 40.1\%-41.5\%) of older adult outpatients received an antibiotic prescription in any study year, and $2.7 \%, 1.7 \%$ and $0.8 \%$ were prescribed an antiviral, antifungal and antiparasitic, respectively. The proportion of older adults prescribed an antiviral increased from $1.9 \%$ in 2006 to $4.4 \%$ in $2015(p<0.001)$; antimicrobial use was otherwise stable across the 10 study years (Figure 1). 


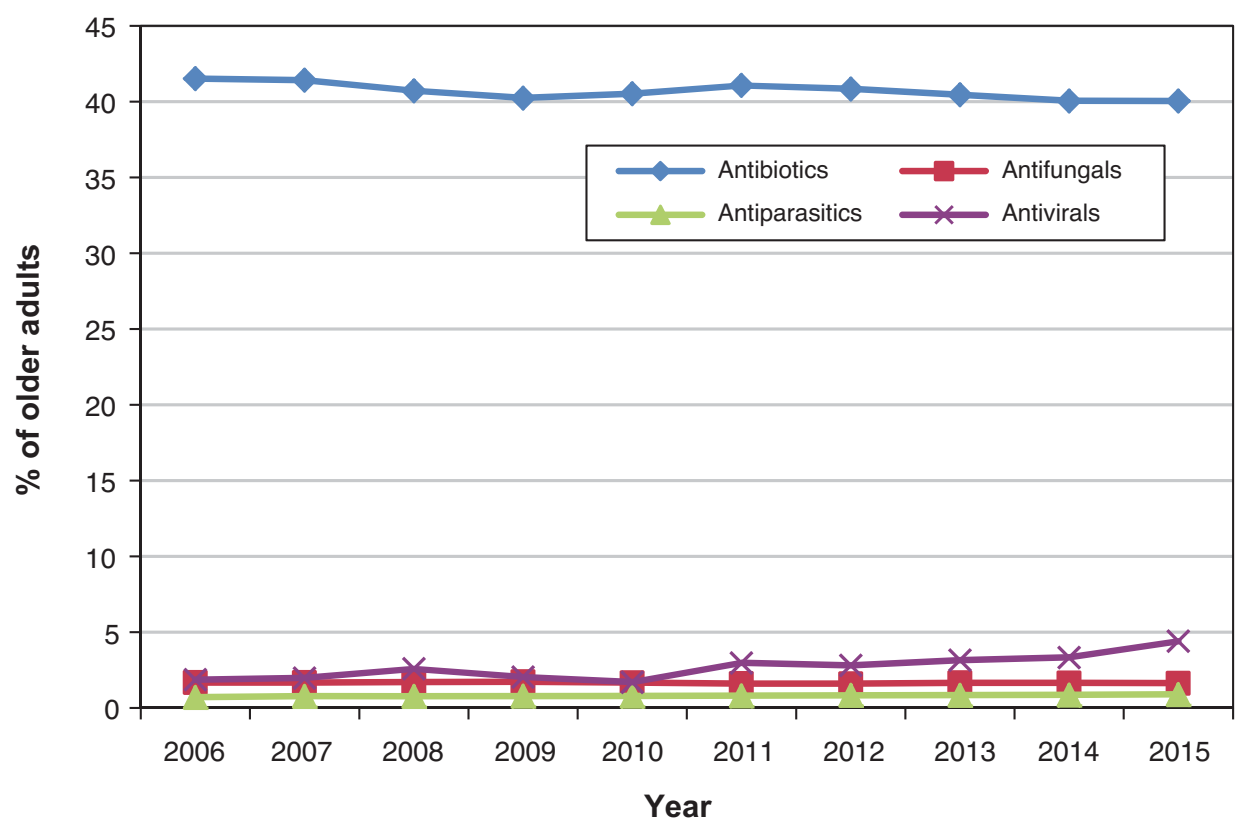

Figure 1: Proportion of Ontario residents aged 65 years or more who received at least 1 outpatient antimicrobial prescription, 2006-2015, by antimicrobial class.

Prescriptions for antibiotics, antifungals and antivirals increased with increasing age. In 2015, 142569 residents $(49.2 \%)$ aged 85 years or more were prescribed an antibiotic, compared to $242555(34.2 \%)$ of those aged 65-69 $(p<0.001)$. Similar increases between the 2 age strata were seen for antivirals (42 $511[14.7 \%]$ v. $12487[1.8 \%], p<0.001)$ and antifungals $(6354[2.2 \%]$ v. $8886[1.3 \%], p<0.001)$.

\section{Antibiotic prescriptions}

The average quantity of antibiotics prescribed per year was 25.1 (range 24.1-25.6) defined daily doses per 1000 persondays. Antibiotic use remained relatively stable between 2006 and 2015, decreasing slightly from 25.6 to 24.1 defined daily doses per 1000 person-days.

The 5 most frequently prescribed antibiotic classes were penicillins, fluoroquinolones, macrolides, cephalosporins and urinary anti-infectives. Trends in their use are shown in Figure 2. Prescriptions for penicillins increased from 6.1 to 7.8 defined daily doses per 1000 person-days between 2006 and 2015. Prescriptions for fluoroquinolones and macrolides decreased over the study period, whereas cephalosporin and urinary anti-infective use remained stable. The rise in penicillin prescriptions was driven by greater use of amoxicillin, from 4.7 to 5.7 defined daily doses per 1000 person-days, and of amoxicillin-clavulanic acid, from 0.68 to 1.8 defined daily doses per 1000 person-days (Appendix 3, available at www. cmajopen.ca/content/5/4/E878/supp1/DC1). Among fluoroquinolones, use of both ciprofloxacin and moxifloxacin declined; among macrolides, clarithromycin use decreased, whereas azithromycin use increased. Nitrofurantoin accounted for most urinary anti-infectives.

\section{Indications for antibiotic prescriptions}

Over the study period, $65.7 \%$ of antibiotics prescribed to older adults did not have a corresponding infectious disease diagnosis detectable within 7 days of the prescription (Appendix 3, white bar segments). The most frequently identified diagnosis was upper respiratory tract infection, associated with $18.9 \%$ of prescriptions, followed by urinary tract infection (6.2\%), skin/softtissue infection $(4.3 \%)$, lower respiratory tract infection $(4.2 \%)$ and other infection (1.2\%). Upper respiratory tract infection was the most common diagnosis associated with penicillins and macrolides, and urinary tract infection was most common with nitrofurantoin and trimethoprim-sulfamethoxazole. Among fluoroquinolones, ciprofloxacin was associated with urinary tract infection, and moxifloxacin was associated with upper and lower respiratory tract infections. For cephalosporins, skin/softtissue infections were the most common diagnosis associated with cephalexin, and upper respiratory tract infection was most common with cefuroxime (Appendix 3).

\section{Antibiotics prescribed for infectious disease diagnoses}

Upper respiratory tract infection was the most common diagnosis associated with an antibiotic prescription. There were 184667 upper respiratory tract infections associated with antibiotic prescriptions in 2006; this increased to 211549 prescriptions in 2015. Increased use of amoxicillin ( 0.80 to 0.98 defined daily doses per 1000 person-days) and amoxicillin-clavulanic 


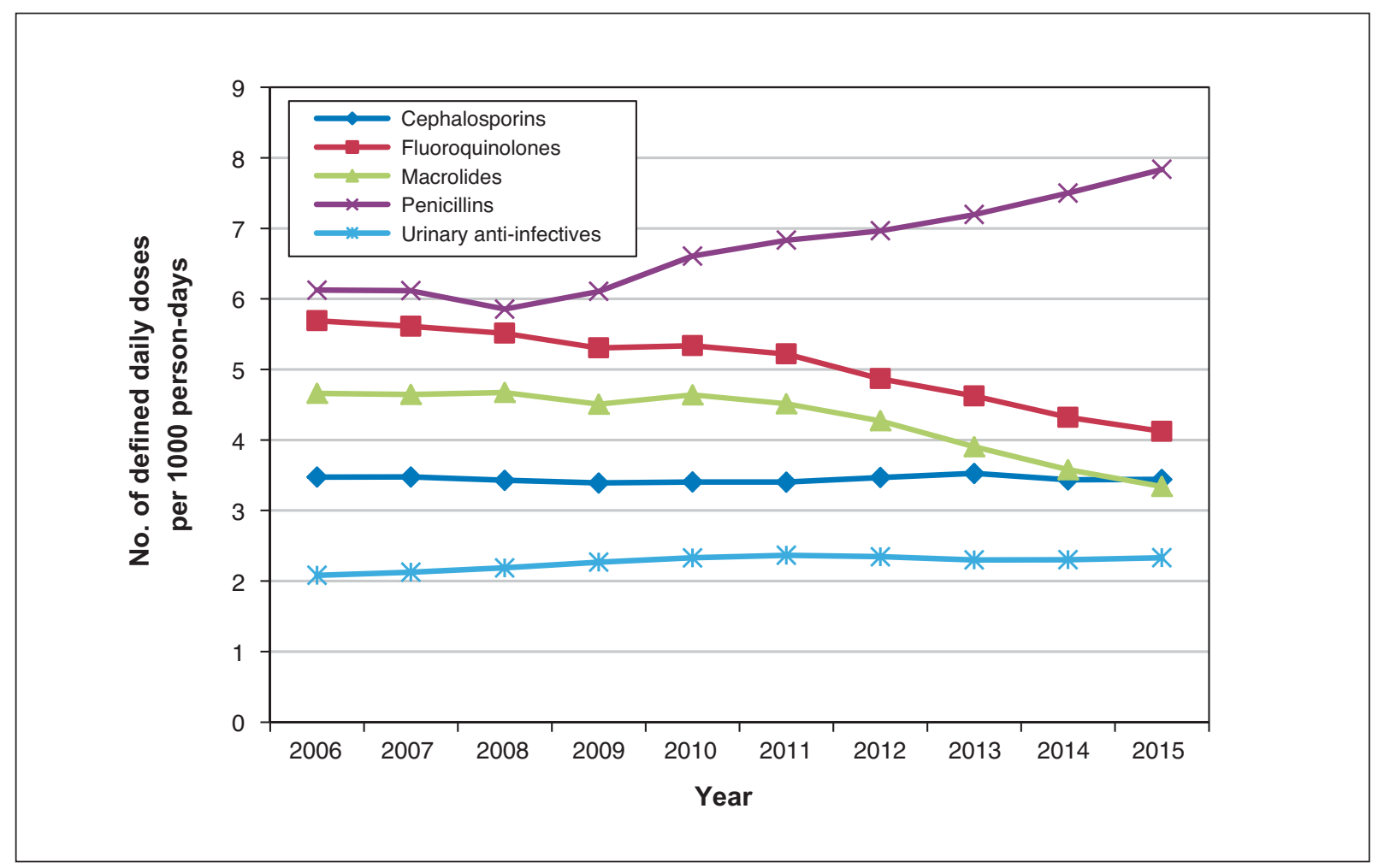

Figure 2: Total outpatient prescriptions, in defined daily doses per 1000 person-days, of the 5 most commonly prescribed antibiotic classes, 2006-2015.

acid (0.10 to 0.29 defined daily doses per 1000 person-days), and decreased use of clarithromycin (1.10 to 0.53 defined daily doses per 1000 person-days), moxifloxacin (0.18 to 0.13 defined daily doses per 1000 person-days) and levofloxacin (0.16 to 0.13 defined daily doses per 1000 person-days) were observed over the study period (Figure 3, A).

Urinary tract infections were the second most common indication for outpatient antibiotics: 75645 antibiotic prescriptions for this diagnosis were identified in 2006, and 100648 were identified in 2015. Use of nitrofurantoin increased from 0.27 to 0.34 defined daily doses per 1000 person-days between 2006 and 2015. Prescriptions for fluoroquinolones declined over the study period, driven largely by a decrease in norfloxacin use, from 0.21 to 0.07 defined daily doses per 1000 person-days; ciprofloxacin use increased slightly. Likewise, prescriptions for trimethoprim-sulfamethoxazole decreased, from 0.22 to 0.14 defined daily doses per 1000 person-days (Figure 3, B).

Lower respiratory tract infection was the third most common indication for outpatient antibiotic treatment in this population: 48408 lower respiratory tract infections were associated with antibiotic prescriptions in 2006, and 66273 in 2015. A substantial decrease in macrolide use was observed. Clarithromycin was the most frequently prescribed antibiotic in 2006, but use declined substantially in 2015 (0.29 to 0.15 defined daily doses per 1000 person-days). In contrast, prescriptions for amoxicillin ( 0.05 to 0.14 defined daily doses per 1000 person-days) and amoxicillin-clavulanic acid (0.03 to 0.13 defined daily doses per 1000 person-days) increased.
There were small declines in prescriptions for fluoroquinolones, although levofloxacin was the most commonly prescribed antibiotic for this indication in 2015 (Figure 3, C).

Last, 45453 antibiotic treatments were prescribed for skin/ soft-tissue infections in 2006, and 64882 in 2015. Cephalexin was the most commonly prescribed antibiotic in both years, increasing from 0.36 to 0.47 defined daily doses per 1000 person-days between 2006 and 2015. Cloxacillin, ciprofloxacin and clarithromycin were less commonly prescribed in 2015 than in 2006, whereas use of clindamycin and amoxicillinclavulanic acid increased between the 2 years (Figure 3, D).

\section{Responsible prescriber}

Family physicians accounted for most antibiotic prescriptions to older adult outpatients (Table 1). Antibiotic prescriptions by both family physicians and specialists increased from 2006 to 2015 , with proportionately more specialist prescriptions in 2015.

\section{Interpretation}

This study of 2879779 older residents of Ontario showed that ambulatory antimicrobial use remained stable from 2006 to 2015. Two-thirds of antibiotic prescriptions did not have a corresponding infectious disease diagnosis within 7 days of the prescription.

We observed increasing use of amoxicillin and amoxicillinclavulanic acid, and decreasing use of fluoroquinolones and 
A. Upper respiratory tract infection

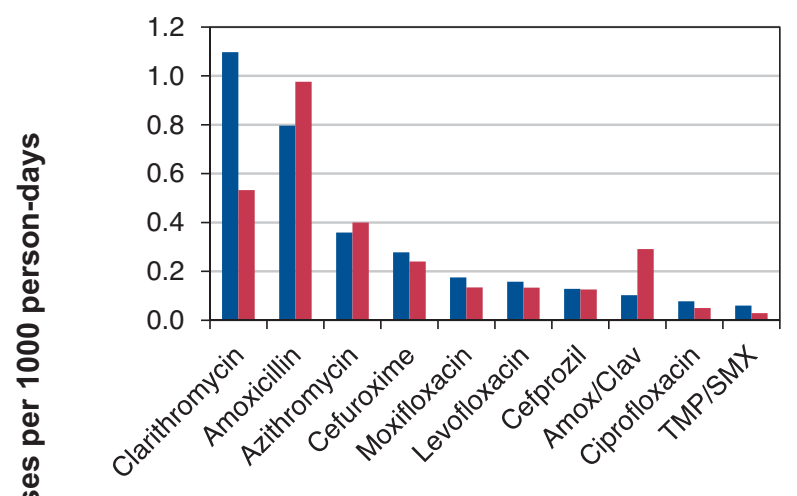

C. Lower respiratory tract infection

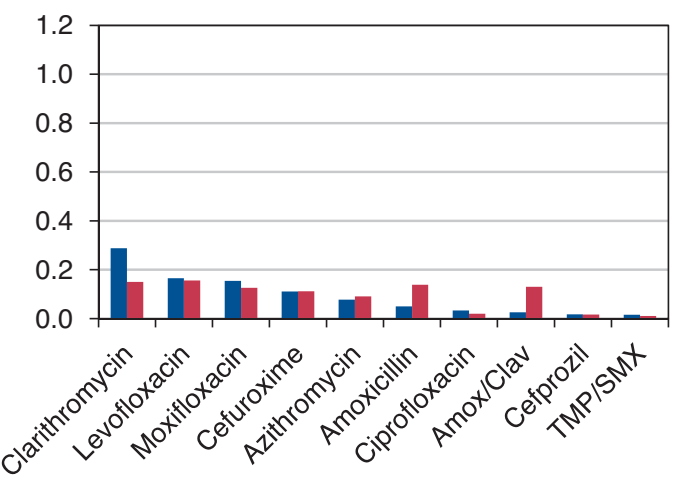

B. Urinary tract infection

$2006 \square 2015$

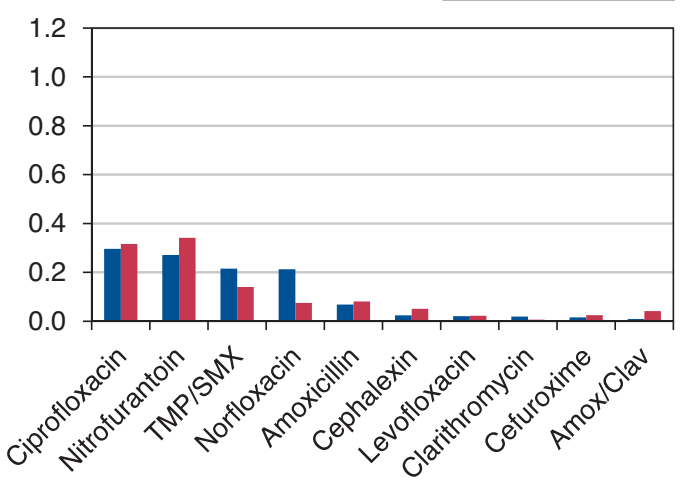

\section{Cellulitis}

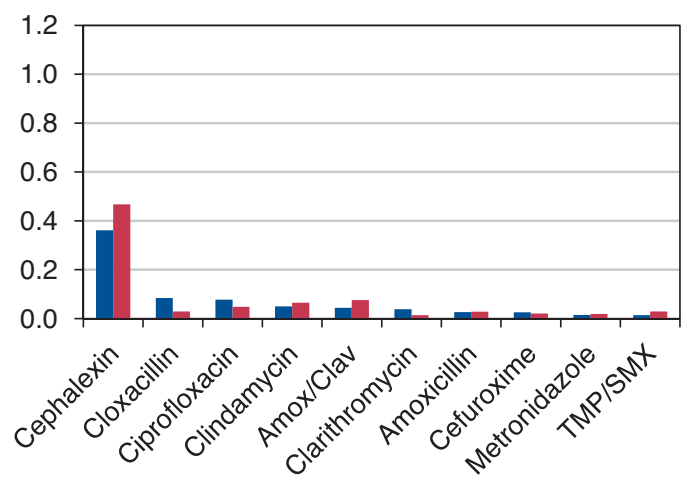

Antibiotic

Figure 3: Outpatient prescriptions, in defined daily doses per 1000 person-days, of the 10 antibiotics most commonly prescribed for (A) upper respiratory tract infections, (B) urinary tract infections, (C) lower respiratory tract infections and (D) skin/soft-tissue infections in 2006 and 2015. Note: $\mathrm{TMP} / \mathrm{SMX}=$ trimethoprim-sulfamethoxazole, Amox/Clav = amoxicillin-clavulanic acid.

macrolides over our study period. These results diverge from the rise in use of broad-spectrum antibiotics reported in prior studies. ${ }^{2,5}, 20$ This may represent a positive change, with physicians favouring narrow-spectrum agents where appropriate. However, overall outpatient antibiotic use was consistent over our study period, averaging 25.1 defined daily doses per 1000 person-days per year, with $40.7 \%$ of older adults receiving a prescription. A 2016 national report on antimicrobial use in Canada showed a similar rate of use in this population (23.0 defined daily doses per 1000 person-days per year) but a higher proportion receiving a prescription (86\%). ${ }^{1}$ Our findings suggest that antibiotic prescribing has not been curtailed by existing interventions. Given that more than $90 \%$ of antimicrobials are prescribed in outpatient care, communitybased efforts are urgently needed, particularly targeting upper respiratory tract and urinary tract infections, the most common diagnostic indications in our study. Interventions should be directed to family physicians, who accounted for more than $70 \%$ of antimicrobial prescriptions to older Ontario residents. However, prescriptions by specialists remain common and rose between 2006 and 2015, with dermatologists and internists previously identified as high-frequency prescribers. ${ }^{1}$

The antibiotics prescribed for upper respiratory tract infections changed over our study period. Fluoroquinolone and macrolide use declined, and penicillin use increased, which contrasts with previous analyses showing rising selection of the former classes. ${ }^{2-4,6,7}$ This is in keeping with guidelines recommending amoxicillin or amoxicillin-clavulanic acid as first-line therapy for bacterial upper respiratory tract infections. ${ }^{40-42}$ However, upper respiratory tract infections were the most common diagnoses associated with antibiotic prescriptions in our study, even though most cases are viral and guidelines discourage routine antibiotic therapy. ${ }^{40-43}$ Other jurisdictions have similarly found high rates of antibiotic use 


\begin{tabular}{|c|c|c|c|c|}
\hline \multirow[b]{2}{*}{ Provider } & \multicolumn{2}{|c|}{ No. $(\%)$ of individual claims; year } & \multicolumn{2}{|c|}{ No. (\%) of defined daily doses; year } \\
\hline & $\begin{array}{c}2006 \\
n=255244\end{array}$ & $\begin{array}{c}2015 \\
n=325981\end{array}$ & $\begin{aligned} & 2006 \\
n= & 2956917\end{aligned}$ & $\begin{array}{c}2015 \\
n=3536835\end{array}$ \\
\hline Family physician & $195745(76.7)$ & $227569(69.8)$ & $2230258(75.4)$ & $2409323(68.1)$ \\
\hline Specialist & 59499 (23.3) & $98412(30.2)$ & $726659(24.6)$ & $1127512(31.9)$ \\
\hline
\end{tabular}

for upper respiratory tract infections..$^{1,5,20}$ This highlights the potential impact a community-based antimicrobial stewardship program could have in reducing overall antibiotic use.

We also found increased penicillin use and decreased macrolide use for lower respiratory tract infections. Although macrolides are recommended as first-line therapy for outpatient community-acquired pneumonia, ${ }^{44}$ resistance rates in Streptococcus pneumoniae have approached $25 \%$ in Canada. ${ }^{1,45,46}$ Thus, macrolides should not be used as monotherapy in this context. The need for "atypical" coverage in treating community-acquired pneumonia has also been questioned. ${ }^{47}$ Within the macrolide class, growing preference for azithromycin over clarithromycin was observed. This finding may be explained by ease of administration, with single daily versus twice-daily dosing, as well as studies showing equivalency between 3-day courses of azithromycin and longer courses of clarithromycin. ${ }^{48,49}$ Another reason for the preference for azithromycin over clarithromycin may be greater awareness of drug interactions involving cytochrome $\mathrm{P} 450$, with CYP3A4 being inhibited by clarithromycin but not azithromycin. ${ }^{50}$

For urinary tract infections, use of nitrofurantoin increased. This is in accordance with guidelines recommending this agent as first-line therapy for urinary tract infections owing to high susceptibility rates and low risk to host flora. ${ }^{51}$ In contrast, use of trimethoprim-sulfamethoxazole declined. This may reflect increasing recognition of its adverse effects, ${ }^{52}$ particularly among older patients coprescribed common cardiovascular and renal medications. ${ }^{37,53,54}$ Prescriptions for ciprofloxacin decreased as well, which may be owing to rising awareness of the risks associated with fluoroquinolones. ${ }^{55-58}$

Cephalexin was consistently the most commonly prescribed antibiotic for skin/soft-tissue infections. Use of clindamycin and amoxicillin-clavulanic acid increased over the study period, which may reflect a change in practice to cover anaerobic organisms as well as methicillin-resistant Staphylococcus aureus in the case of clindamycin. However, clindamycin is associated with the highest risk of Clostridium difficile infection among antibiotics..$^{59,60}$

The fact that two-thirds of antibiotics prescribed were not associated with a recorded infectious disease diagnosis suggests that our databases were unable to capture most antibiotic indications, even in the context of a universal single-payer health care system, access to linkable physician claim, hospital discharge, same-day surgery and emergency department databases, and the use of a broad 7-day window around prescrip- tions to identify diagnoses. Effective surveillance of community antibiotic use will require more comprehensive methods of capturing antibiotic indication, such as linkage to electronic medical records and mandatory reporting of diagnosis with each prescription.

\section{Limitations}

Antibiotic prescriptions and infectious disease diagnoses were linked through their presence within 7 days of one another. Although these antibiotic-diagnosis associations are likely accurate given their temporal proximity, we could not ascertain causation. We identified diagnoses using infectiousdisease-specific diagnostic codes; broader systems-based codes may have improved our linkage rates. For inpatient diagnoses, captured in the Discharge Abstracts Database, date of admission was taken as the date of diagnosis. Diagnoses around outpatient antibiotic prescriptions, and vice versa, may consequently have been missed, particularly in cases long hospital stays and infections fully treated in hospital. Furthermore, the Ontario Health Insurance Plan database allows for only a single diagnosis to be recorded in each claim; infectious disease diagnoses may have been unrecorded in visits involving multiple diagnoses. Another limitation is our categorization of diagnoses. Disease-specific diagnoses, such as acute bronchitis and acute rhinosinusitis, would have allowed us to better assess antimicrobial appropriateness. Last, defined daily dose may be an inaccurate measure of drug use in patients with renal impairment, a common comorbidity in older populations.

\section{Conclusion}

In our analysis of outpatient antimicrobial use among older adults in Ontario, we observed a trend toward greater selection of narrow-spectrum antibiotics. However, total use was stable from 2006 to 2015, and antibiotics were frequently prescribed for upper respiratory tract infections. This emphasizes that misuse and overuse of antibiotics remain a problem. Interventions to improve antibiotic prescribing in ambulatory care are therefore warranted. In addition, two-thirds of antibiotic prescriptions were not associated with an infectious disease diagnosis, which shows that existing methods of surveillance in Ontario are not capable of determining antibiotic indication. Given the lack of information on outpatient antibiotic use in Canada, efforts to monitor the quantity, composition and appropriateness of community use need to be 
strengthened. Our results can be used to guide such efforts and to benchmark antimicrobial stewardship interventions among outpatients.

\section{References}

1. Canadian Antimicrobial Resistance Surveillance System Report 2016. Ottawa: Public Health Agency of Canada; 2016.

2. Steinman MA, Gonzales R, Linder JA, et al. Changing use of antibiotics in community-based outpatient practice, 1991-1999. Ann Intern Med 2003;138: $525-33$.

3. Grijalva CG, Nuorti JP, Griffin MR. Antibiotic prescription rates for acute respiratory tract infections in US ambulatory settings. 7AMA 2009;302:758-66.

4. Fairlie T, Shapiro DJ, Hersh AL, et al. National trends in visit rates and antibiotic prescribing for adults with acute sinusitis. Arch Intern Med 2012;172: $1513-4$.

5. Shapiro DJ, Hicks LA, Pavia AT, et al. Antibiotic prescribing for adults in ambulatory care in the USA, 2007-09. F Antimicrob Chemother 2014;69:234-40.

6. Hersh AL, Fleming-Dutra KE, Shapiro DJ, et al. Frequency of first-line antibiotic selection among US ambulatory care visits for otitis media, sinusitis, and pharyngitis. FAMA Intern Med 2016;176:1870-2.

7. Fleming-Dutra KE, Hersh AL, Shapiro DJ, et al. Prevalence of inappropriate antibiotic prescriptions among US ambulatory care visits, 2010-2011. FAMA 2016;315:1864-73.

8. Spellberg B, Guidos R, Gilbert D, et al. The epidemic of antibiotic-resistant infections: a call to action for the medical community from the Infectious Diseases Society of America. Clin Infect Dis 2008;46:155-64.

9. Costelloe C, Metcalfe C, Lovering A, et al. Effect of antibiotic prescribing in primary care on antimicrobial resistance in individual patients: systematic review and meta-analysis. BM7 2010;340:c2096.

10. Hicks LA, Chien YW, Taylor TH, et al. Outpatient antibiotic prescribing and nonsusceptible Streptococcus pneumoniae in the United States, 1996-2003. Clin Infect Dis 2011;53:631-9.

11. Goossens H, Ferech M, Vander Stichele R, et al. Outpatient antibiotic use in Europe and association with resistance: a cross-national database study. Lancet 2005:365:579-87.

12. van de Sande-Bruinsma N, Grundmann H, Verloo D, et al. Antimicrobial drug use and resistance in Europe. Emerg Infect Dis 2008;14:1722-30.

13. Dagan R, Barkai G, Givon-Lavi N, et al. Seasonality of antibiotic-resistant Streptococcus pneumoniae that causes acute otitis media: A clue for an antibioticrestriction policy? F Infect Dis 2008;197:1094-102.

14. Dantes R, Mu Y, Hicks LA, et al. Association between outpatient antibiotic prescribing practices and community-associated Clostridium difficile infection. Open Forum Infect Dis 2015;2:ofv113.

15. Shehab N, Patel PR, Srinivasan A, et al. Emergency department visits for antibiotic-associated adverse events. Clin Infect Dis 2008;47:735-43.

16. Suda KJ, Hicks LA, Roberts RM, et al. A national evaluation of antibiotic expenditures by healthcare setting in the United States, 2009. F Antimicrob Chemother 2013;68:715-8.

17. Smith R, Coast J. The true cost of antimicrobial resistance. BMf 2013;346: f1493.

18. Wang A, Daneman N, Tan C, et al. Evaluating the relationship between hospital antibiotic use and antibiotic resistance in common nosocomial pathogens. Infect Control Hosp Epidemiol 2017;38:1457-63.

19. Sun L, Klein EY, Laxminarayan R. Seasonality and temporal correlation between community antibiotic use and resistance in the United States. Clin Infect Dis 2012;55:687-94.

20. Lee GC, Reveles KR, Attridge RT, et al. Outpatient antibiotic prescribing in the United States: 2000 to 2010. BMC Med 2014;12:96.

21. Haeseker MB, Dukers-Muijrers NH, Hoebe CJ, et al. Trends in antibiotic prescribing in adults in Dutch general practice. PLoS One 2012;7:e51860.

22. Norris P, Horsburgh S, Keown S, et al. Too much and too little? Prevalence and extent of antibiotic use in a New Zealand region. 7 Antimicrob Chemother 2011;66:1921-6.

23. Pan A, Buttazzi R, Marchi M, et al. Secular trends in antibiotic consumption in the adult population in Emilia-Romagna, Italy, 2003-2009. Clin Microbiol Infect 2011;17:1698-703.

24. 2015/16 report card for the Ontario Drug Benefit Program. Toronto: Ontario Ministry of Health and Long-Term Care; 2016.

25. Faulkner CM, Cox HL, Williamson JC. Unique aspects of antimicrobial use in older adults. Clin Infect Dis 2005;40:997-1004.

26. Beckett CL, Harbarth S, Huttner B. Special considerations of antibiotic prescription in the geriatric population. Clin Microbiol Infect 2015;21:3-9.

27. Denkinger CM, Grant AD, Denkinger M, et al. Increased multi-drug resistance among the elderly on admission to the hospital - a 12-year surveillance study. Arch Gerontol Geriatr 2013;56:227-30.

28. Pop-Vicas A, Tacconelli E, Gravenstein S, et al. Influx of multidrug-resistant, gram-negative bacteria in the hospital setting and the role of elderly patients with bacterial bloodstream infection. Infect Control Hosp Epidemiol 2009;30: $325-31$.
29. March A, Aschbacher R, Dhanji H, et al. Colonization of residents and staff of a long-term-care facility and adjacent acute-care hospital geriatric unit by multiresistant bacteria. Clin Microbiol Infect 2010;16:934-44.

30. Born KB, Leis JA, Gold WL, et al. "Choosing Wisely Canada" and antimicrobial stewardship: a shared focus on reducing unnecessary care. Can Commun Dis Rep 2015;41:9-13.

31. AntibioticAwareness.ca. Antibiotic Awareness Week in Canada: Nov 13-19, 2017. Winnipeg: National Collaborating Centre for Infectious Diseases; 2016. Available: https://nccid.ca/antibiotic-awareness/ (accessed 2017 Mar. 16).

32. Drekonja DM, Filice GA, Greer N, et al. Antimicrobial stewardship in outpatient settings: a systematic review. Infect Control Hosp Epidemiol 2015;36:142-52.

33. Patrick D, Grant J, Saxinger L. Surveillance of antimicrobial resistance and utilization in Canada. Winnipeg: National Collaborating Centre for Infectious Diseases; 2014.

34. Levy AR, O'Brien BJ, Sellors C, et al. Coding accuracy of administrative drug claims in the Ontario Drug Benefit database. Can 7 Clin Pharmacol 2003;10:67-71.

35. Juurlink D, Preyra C, Croxford R, et al. Canadian Institute for Health Information Discharge Abstract Database: a validation study. Toronto: Institute for Clinical Evaluative Sciences; 2006.

36. Daneman N, Gruneir A, Bronskill SE, et al. Prolonged antibiotic treatment in long-term care: role of the prescriber. FAMA Intern Med 2013;173:673-82.

37. Fralick M, Macdonald EM, Gomes T, et al. Co-trimoxazole and sudden death in patients receiving inhibitors of renin-angiotensin system: population based study. BM7 2014;349:g6196.

38. Daneman N, Bronskill SE, Gruneir A, et al. Variability in antibiotic use across nursing homes and the risk of antibiotic-related adverse outcomes for individual residents. FAMA Intern Med 2015;175:1331-9.

39. Definition and general considerations. Oslo: WHO Collaborating Centre for Drug Statistics Methodology; 2009. Available: www.whocc.no/ddd/definition and_general_considera/ (accessed 2015 Aug. 17).

40. Harris AM, Hicks LA, Qaseem A; High-Value Care Task Force of the American College of Physicians and the Centers for Disease Control and Prevention. Appropriate antibiotic use for acute respiratory tract infection in adults: advice for High-Value Care from the American College of Physicians and the Centers for Disease Control and Prevention. Ann Intern Med 2016;164:425-34

41. Wong DM, Blumberg DA, Lowe LG. Guidelines for the use of antibiotics in acute upper respiratory tract infections. Am Fam Physician 2006;74:956-66.

42. Chow AW, Benninger MS, Brook I, et al. IDSA clinical practice guideline for acute bacterial rhinosinusitis in children and adults. Clin Infect Dis 2012;54: e72-112.

43. Desrosiers M, Evans GA, Keith PK, et al. Canadian clinical practice guidelines for acute and chronic rhinosinusitis. 7 Otolaryngol Head Neck Surg 2011;40 (Suppl 2):S99-193.

44. Mandell LA, Wunderink RG, Anzueto A, et al. Infectious Diseases Society of America/American Thoracic Society consensus guidelines on the management of community-acquired pneumonia in adults. Clin Infect Dis 2007;44(Suppl 2): S27-72.

45. Demczuk W, Griffith A, Singh R, et al. National laboratory surveillance of invasive streptococcal disease in Canada - annual summary 2013. Ottawa: Public Health Agency of Canada; 2013.

46. Wierzbowski AK, Karlowsky JA, Adam HJ, et al. Evolution and molecular characterization of macrolide-resistant Streptococcus pneumoniae in Canada between 1998 and 2008. 7 Antimicrob Chemother 2014;69:59-66.

47. Postma DF, van Werkhoven CH, van Elden LJ, et al. Antibiotic treatmen strategies for community-acquired pneumonia in adults. N Engl 7 Med 2015; 372:1312-23.

48. Drehobl MA, De Salvo MC, Lewis DE, et al. Single-dose azithromycin microspheres vs clarithromycin extended release for the treatment of mild-to-moderate community-acquired pneumonia in adults. Chest 2005;128:2230-7.

49. O'Doherty B, Muller O. Randomized, multicentre study of the efficacy and tolerance of azithromycin versus clarithromycin in the treatment of adults with mild to moderate community-acquired pneumonia. Azithromycin Study Group. Eur 7 Clin Microbiol Infect Dis 1998;17:828-33.

50. Pai MP, Graci DM, Amsden GW. Macrolide drug interactions: an update. Ann Pharmacother 2000;34:495-513.

51. Gupta K, Hooton TM, Naber KG, et al. International clinical practice guidelines for the treatment of acute uncomplicated cystitis and pyelonephritis in women: a 2010 update by the Infectious Diseases Society of America and the European Society for Microbiology and Infectious Diseases. Clin Infect Dis 2011:52:e103-20.

52. Ho JM, Juurlink DN. Considerations when prescribing trimethoprimsulfamethoxazole. CMA7 2011;183:1851-8.

53. Antoniou T, Gomes T, Juurlink DN, et al. Trimethoprim-sulfamethoxazoleinduced hyperkalemia in patients receiving inhibitors of the renin-angiotensin system: a population-based study. Arch Intern Med 2010;170:1045-9.

54. Antoniou T, Hollands S, Macdonald EM, et al. Trimethoprim-sulfamethoxazole and risk of sudden death among patients taking spironolactone. CMA7 2015;187: E138-43.

55. Khaliq Y, Zhanel GG. Fluoroquinolone-associated tendinopathy: a critical review of the literature. Clin Infect Dis 2003;36:1404-10.

56. Daneman N, Lu H, Redelmeier DA. Fluoroquinolones and collagen associated severe adverse events: a longitudinal cohort study. BMF Open 2015;5:e010077. 
57. Lee CC, Lee MT, Chen YS, et al. Risk of aortic dissection and aortic aneurysm in patients taking oral fluoroquinolone. 7AMA Intern Med 2015;175: 1839-47.

58. Etminan M, Brophy JM, Samii A. Oral fluoroquinolone use and risk of peripheral neuropathy: a pharmacoepidemiologic study. Neurology 2014;83:1261-3.

59. Brown KA, Khanafer N, Daneman N, et al. Meta-analysis of antibiotics and the risk of community-associated Clostridium difficile infection. Antimicrob Agents Chemother 2013;57:2326-32.

60. Deshpande A, Pasupuleti V, Thota P, et al. Community-associated Clostridium difficile infection and antibiotics: a meta-analysis. 7 Antimicrob Chemother 2013; 68:1951-61.

Affiliations: Sunnybrook Research Institute (Tan, Daneman) and Division of Infectious Diseases (Daneman), Sunnybrook Health Sciences Centre; Institute for Clinical Evaluative Sciences (Graves, Lu, Chen, Li, Schwartz, Daneman); Public Health Ontario (Schwartz, Daneman); Dalla Lana School of Public Health (Schwartz), University of Toronto, Toronto, Ont.

Contributors: Nick Daneman, Hong Lu and Erin Graves conceptualized and designed the study, and developed the statistical analysis plan. Nick Daneman and Charlie Tan analyzed and interpreted the data and drafted the manuscript. Hong Lu, Anna Chen and Shudong Li contributed to data acquisition and analysis. Kevin Schwartz contributed to data interpretation and manuscript revision. All of the authors gave final approval of the version to be published and agreed to be accountable for all aspects of the work.

Funding: This work was supported by a Canadian Institutes of Health Research Clinician Scientist Salary Award to Nick Daneman and an Applied Health Research Question fund via the Institute for Clinical Evaluative Sciences.

Disclaimer: This study was supported by the Institute for Clinical Evaluative Sciences (ICES), which is funded by an annual grant from the Ontario Ministry of Health and Long-Term Care (MOHLTC). The opinions, results and conclusions reported in this article are those of the authors and are independent from the funding sources. No endorsement by the ICES or the Ontario MOHLTC is intended or should be inferred.

Supplemental information: For reviewer comments and the original submission of this manuscript, please see www.cmajopen.ca/content/5/4/ E878/suppl/DC1. 